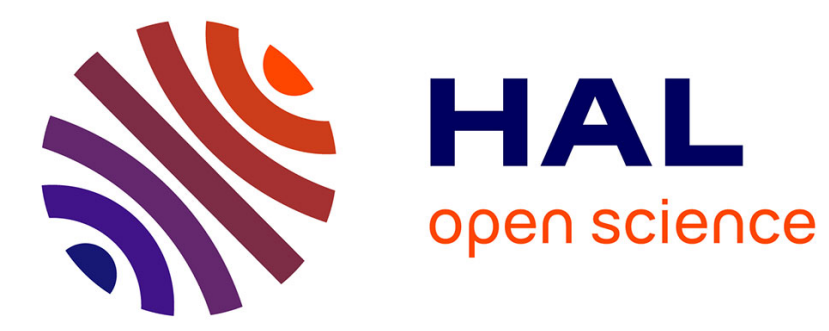

\title{
Anisotropic Mullins stress softening of a deformed silicone holey plate
}

Marie Rebouah, Guilherme Machado, Grégory Chagnon, Denis Favier

\section{To cite this version:}

Marie Rebouah, Guilherme Machado, Grégory Chagnon, Denis Favier. Anisotropic Mullins stress softening of a deformed silicone holey plate. Mechanics Research Communications, 2013, 49, pp.3643. 10.1016/j.mechrescom.2013.02.002 . hal-01974917

\section{HAL Id: hal-01974917 https://hal.science/hal-01974917}

Submitted on 9 Jan 2019

HAL is a multi-disciplinary open access archive for the deposit and dissemination of scientific research documents, whether they are published or not. The documents may come from teaching and research institutions in France or abroad, or from public or private research centers.
L'archive ouverte pluridisciplinaire HAL, est destinée au dépôt et à la diffusion de documents scientifiques de niveau recherche, publiés ou non, émanant des établissements d'enseignement et de recherche français ou étrangers, des laboratoires publics ou privés. 


\title{
Anisotropic Mullins stress softening of a deformed silicone holey plate
}

\author{
M. Rebouah, G. Machado, G. Chagnon*, D. Favier \\ Université de Grenoble/CNRS, Laboratoire 3SR, Cedex 9, 38041 Grenoble, France.
}

\begin{abstract}
Rubber like materials parts are designed using finite element code in which more and more precise and robust constitutive equations are implemented. In general, constitutive equations developed in literature to represent the anisotropy induced by the Mullins effect present analytical forms that are not adapted to finite element implementation. The present paper deals with the development of a constitutive equation that represents the anisotropy of the Mullins effect using only strain invariants. The efficiency of the modeling is first compared to classical homogeneous experimental tests on a filled silicone rubber. Second, the model is tested on a complex structure. In this aim, a silicone holey plate is molded and tested in tension, its local strain fields are evaluated by means of digital image correlation. The experimental results are compared to the simulations from the constitutive equation implemented in a finite element code. Global measurements (i.e. force and displacement) and local strain fields are successfully compared to experimental measurements to validate the model.
\end{abstract}

Key words: Mullins effect; stress-softening; finite element implementation; constitutive equation

\section{Introduction}

Both natural and synthetic elastomers are widely used in industrial design. Numerical simulations are often used to develop new parts. These simulations are fundamental in the optimization process of parts design. One of the main difficulties of the engineers is the choice of an adapted constitutive equation able to represent the behavior of the rubber like materials. The choice is often oriented towards an hyperelastic constitutive equation requiring an energy density. Finite element codes present a large choice of strain energy densities forms, even if the Mooney (1940) constitutive equation stays one of the most used.

However rubber like materials present a lot of other phenomena than pure hyperelasticity to take into account in their behavior, as for example: the Mullins effect, the hysteretis and time dependent behavior. The Mullins effect can be very important as the mechanical behavior of the material can totally change after a first loading. For some rubber parts, it is very important to take into account the Mullins effect, but very few constitutive equations are implemented in industrial finite element codes. Eventhough, many researchers have developed isotropic constitutive equations for the Mullins effect and proposed a finite element implementation (see for example Cantournet et al. (2009); Chagnon et al. (2006); Gracia et al. (2009); Guo et al. (2006)).

\footnotetext{
${ }^{*}$ Corresponding author

Email address: gregory.chagnon@grenoble-inp.fr (G. Chagnon)

Preprint submitted to Mechanic Research Communications
}

October 19, 2012 
Another important point is that, many new experimental data are proposed in the literature to emphasize that the Mullins effect is strongly anisotropic (Muhr et al., 1999; Park and Hamed, 2000; Pawelski, 2001; Besdo and Ihlemann, 2003; Laraba-Abbes et al., 2003; Diani et al., 2006a; Hanson et al., 2005; Itskov et al., 2006; Machado et al., 2012b; Dorfmann and Pancheri, 2012). Different constitutive equations have been proposed but they are not adapted to finite element implementation. The only formulation implemented in a finite element code was proposed by Göktepe and Miehe (2005) who extended the approach of Miehe et al. (2004). This model presents good results, but its formulation makes difficult its numerical use into finite element codes, as it needs complex algorithm implementation and also some linearizations (see for example Miehe and Keck (2000); Kaliske (2000)). A formulation written with strain invariants permits to avoid such technics and ensure a better convergence. Some constitutive equations has been developed for living tissues (Peña et al., 2009; Bose and Dorfmann, 2009; Kroon and Holzapfel, 2008) but they are limited to materials presenting two reinforced directions. In this way, in this paper, a new anisotropic model based on strain invariant formulation, is proposed and implemented in a finite element code.

Recently, Machado et al. (2010, 2012b) developed a large database for a filled silicone rubber including on one hand cyclic classical experimental tests and on the other hand uniaxial tests realized after different uniaxial and biaxial tension tests. This database is, here, used to build a new constitutive equation easily implementable in finite element codes. In Section 2, the constitutive equation is detailled and the ability of the model to describe recent experimental data is discussed. In Section 3, the subject of the numerical implementation of the model is treated. In Section 4, the ability of the model to describe complex structures is tackled by means of a specific test on a rectangular plate containing five holes. The global and local estimations of the model are compared to experimental measures. Finally, Section 5 contains some concluding remarks of the modeling.

\section{Anisotropic modeling of the Mullins effect}

\subsection{General formulation in strain invariants}

Different anisotropic approaches to model Mullins effect were proposed in literature, but none of them was only expressed in term of strain invariants. Shariff (2006) and Itskov et al. (2010) took into account three principal damage directions to reproduce a special behavior in the direction orthogonal to loading. In a more general way, the spatial repartition of Bazant and Oh (1986) was used by many authors to create an anisotropic model. Diani et al. (2006b) and Dargazany and Itskov (2009) generalized the network evolution proposed by Marckmann et al. (2002) to an anisotropic approach by taking into account the maximum elongation in each spatial direction. Later Merckel et al. $(2011,2012)$ introduced a new framework and proposed a softening anisotropic criterion adapted to complex loading states.

The stress softening phenomenon has been often associated to the presence of fillers in the rubber, but Harwood et al. (1965) showed that stress softening can also occur in unfilled rubber, even if it is reduced compared to filled rubber. For silicone rubbers, Meunier et al. (2008) observed no Mullins effect for an unfilled one, whereas Machado et al. (2010, 2012b) observed stress softening for a filled one. As a consequence, it can be considered that fillers in silicone rubbers are mainly responsible of the Mullins effect. Thus, as proposed by Govindjee and Simo (1992) the strain energy density $\mathcal{W}$ is additively decomposed into two parts: one that represents the energy density of the chains linked to other chains $\mathcal{W}_{c c}$ and an other part that represents the energy density of the chains linked to filler $\mathcal{W}_{c f}$, the total strain energy density is $\mathcal{W}=\mathcal{W}_{c c}+\mathcal{W}_{c f}$. It is considered that 
only $\left(\mathcal{W}_{c f}\right)$ can evolve with the Mullins effect. As a consequence $\mathcal{W}_{c c}$ is represented by a classical hyperelastic isotropic energy density and $\mathcal{W}_{c f}$ must be represented by an anisotropic strain energy that can evolve with the deformation history of the material. The ideal representation would be to propose a full integration of all spatial directions as proposed by Wu and Van der Giessen (1993) in hyperleasticity, but it is not adapted to finite element implementation. A spatial discretization is needed. Forty-two initial spatial directions, noted $\mathbf{A}^{(i)}$, are introduced, these directions are those proposed by Bazant and Oh (1986). Therefore, the strain energy density is written as:

$$
\mathcal{W}=\mathcal{W}_{c c}\left(I_{1}, I_{2}\right)+\sum_{i=1}^{n} \omega^{(i)} \mathcal{F}^{(i)} \mathcal{W}_{c f}^{(i)}\left(I_{4}^{(i)}\right)
$$

where $I_{1}, I_{2}$ are the first and second strain invariants of the right Cauchy-Green strain tensor $\mathbf{C}$. The strain in each direction is defined by means of $I_{4}^{(i)}=\mathbf{A}^{(i)} \cdot \mathbf{C A}{ }^{(i)} \cdot \omega^{(i)}$ represents the weight of each direction and $\mathcal{F}^{(i)}$ is the Mullins effect evolution function. The initial direction $\mathbf{A}^{(i)}$ are transformed in $\mathbf{a}^{(i)}$ by $\mathbf{a}^{(i)}=\mathbf{F} \mathbf{A}^{(i)}$, where $\mathbf{F}$ is the deformation gradient.

Classicaly, in an isotropic approach, the evolution function $\mathcal{F}^{(i)}$ would be written through the strain energy density, but Chagnon et al. (2004) showed that the first invariant can also be used. In an anisotropic approach, the elongation in each direction is used Diani et al. (2006b), knowing that the elongation is the square root of the invariant $I_{4}$. According to the conclusions of Machado et al. (2012b), it is chosen to describe the stress-softening function according to $I_{1}$ and $I_{4}{ }^{(i)}$. For each direction $(i)$, an evolution function which depends on three terms $\mathcal{F}_{1}, \mathcal{F}_{2}, \mathcal{F}_{3}$ is proposed:

$$
\mathcal{F}=1-\mathcal{F}_{1}\left(I_{1}{ }^{\max }-I_{1}\right) \mathcal{F}_{2}\left(I_{4}{ }^{\max (i)}-I_{4}{ }^{(i)}\right) \mathcal{F}_{3}\left(\frac{I_{4}{ }^{\max (i)}}{I_{4}{ }^{\max }}\right)
$$

Where $I_{1}{ }^{\max }$ and $I_{4}{ }^{\max (i)}$ represent the maximum values taken during the material history by $I_{1}$ and $I_{4}{ }^{(i)}$ respectively. $I_{4}{ }^{\max }=\max _{i}\left(I_{4}{ }^{(i)}\right)$ is the maximum dilatation in space and time. As proposed by Zuñiga and Beatty (2002), a function that is constant during first loading and that evolves with the maximum and current deformations is imposed for the evolution function.

\subsection{A particular form for evolution function}

A Mooney (1940) constitutive equation is chosen for $\left(\mathcal{W}_{c c}\right)$, and a Kaliske (2000) quadratic equation $K\left(I_{4}^{(i)}-1\right)^{2}$ is chosen for $\left.\mathcal{W}_{c f}^{(} i\right)$. A first particular form is proposed for the stress-softening function, considering that a minimum of parameters should be introduced:

$$
\mathcal{F}^{(i)}=1-\eta \sqrt{\frac{I_{1 \max }-I_{1}}{I_{1 \max }-3}}\left(\frac{I_{4 \max }^{(i)}-I_{4}^{(i)}}{I_{4 \max }^{(i)}-1}\right)\left(\frac{I_{4 \max }^{(i)}}{I_{4 \max }}\right)^{4}
$$

In this way, the evolution function depends only on one parameter: $\eta$. The large experimental database proposed by Machado et al. $(2010,2012 \mathrm{~b})$ on a filled silicone rubber is used to fit the model. These experimental results are decomposed into three parts: first the classical uniaxial tension, planar tension and biaxial tests realized by means of a bulge test (Machado et al., 2012a), second the complex tensile tests with change of directions after the first loading; and third biaxial tensile tests followed by uniaxial tensile tests. The three hyperelastic parameters $C_{1}, C_{2}$ and $K^{(i)}$ are obtained by fitting the different first loading curves. The followings values are obtained: 
$C_{1}=0.05 \mathrm{MPa}, C_{2}=0.03 \mathrm{MPa}$ and $\forall i \quad K^{(i)}=0.10 \mathrm{MPa}$. The last parameter is fitted to represent every second loading curves: $\eta=4$.

The simulations of the cyclic uniaxial tensile, pure shear and equibiaxial tensile tests are presented in Fig. 1. It appears that the model describes well the stress softening for all these tests. The model predicts well uniaxial and pure shear tests whereas equibiaxial first loading curve is underestimated. This phenomenon is expected since first loading depends only on the hyperelastic equation. As explained by Marckmann and Verron (2006), it is difficult to fit all the different tests with the same energy density.

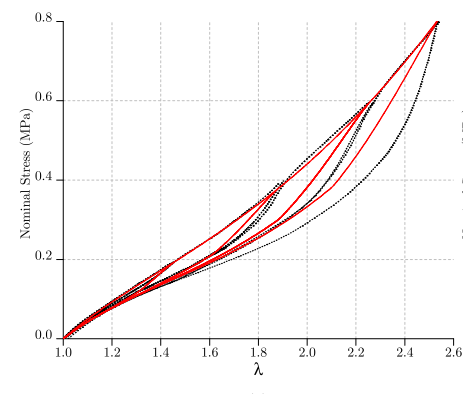

(a)

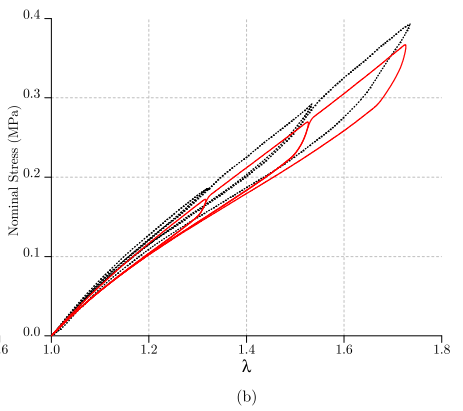

(b)

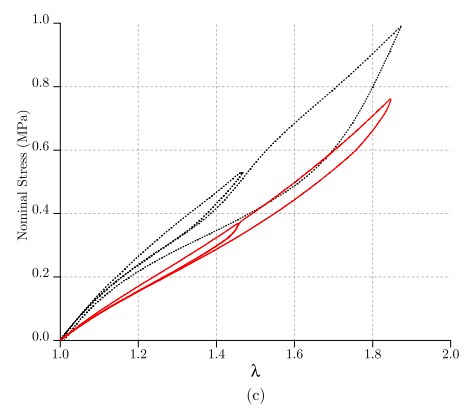

Figure 1: Comparison of the model (solid lines) with experimental data (dotted lines) from Machado et al. (2010) (a) cyclic uniaxial tensile test, (b) cyclic pure shear test (c) cyclic equibiaxial test.

The proposed model is now compared with the experimental data of the two complex preconditioning methods. First, Fig. 2, presents the results for tensile tests with a change of loading direction between the first and second loadings. The results from the model do not superimpose exactly experimental data, but all trends are quite well described for the different directions. Second, the model predictions are compared with biaxial pre-stretching tests results. The biaxial loading is characterized by the biaxiality ratio defined as $\mu=\ln \left(\lambda_{\min }\right) / \ln \left(\lambda_{\max }\right)$ (where $\lambda_{\min }$ and $\lambda_{\max }$ are the minimum and maximum in-plane principal elongation). Tests with different biaxiality ratios were used for the simulation. The comparison of the second loading curves is presented in Fig. 3. It appears that the stress softening is slightly overestimated but the return point on the first loading curve is very well described.

\section{Finite element implementation}

Considering the model proposed in the last section, the strain energy density depends on the first, second and forty-two fourth invariants, i.e., $\mathcal{W}=\mathcal{W}\left(I_{1}, I_{2}, I_{4}^{(i)}\right)$. As the numerical imple- 


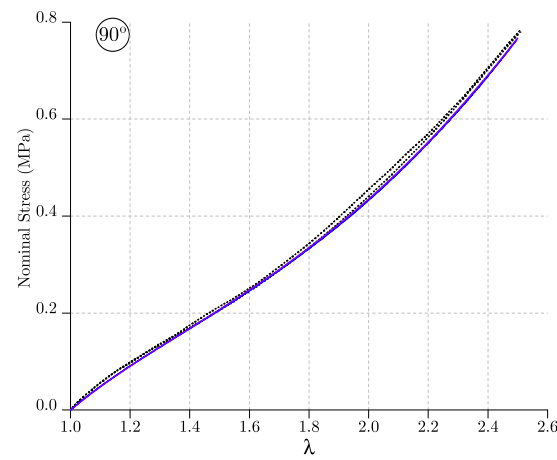

(a)

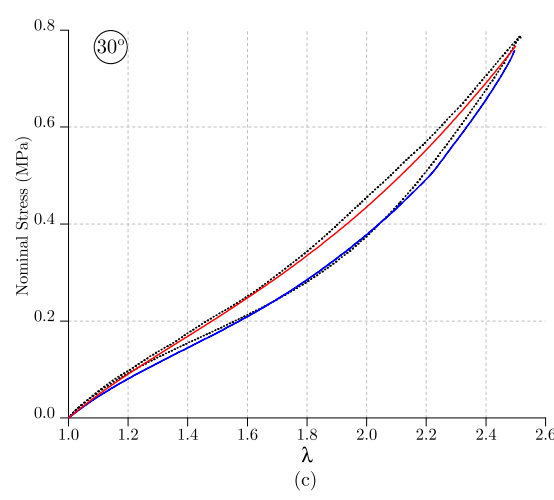

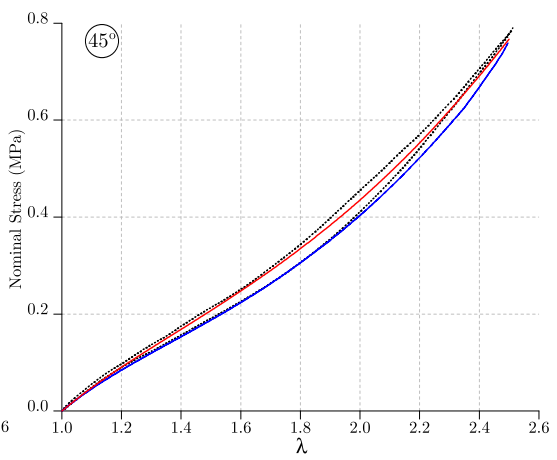

(b)

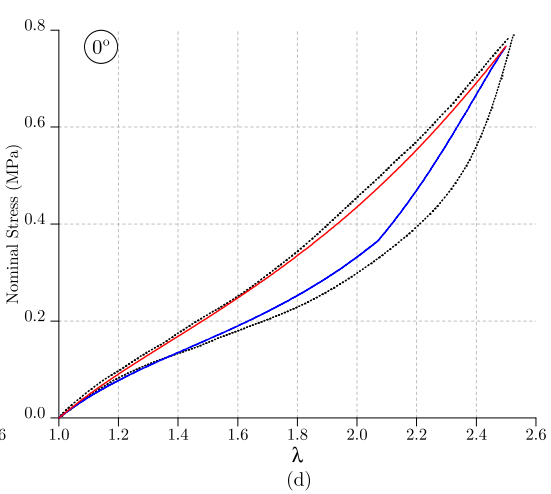

Figure 2: Comparison of the model with uniaxial pre-stretching experimental data (dotted lines) from Machado et al. (2012b). (a) Second tensile test oriented at $90^{\circ}$ (b) Second tensile test oriented at $45^{\circ}$ (e) Second tensile test oriented at $30^{\circ}$ (f) Second tensile test oriented at $0^{\circ}$

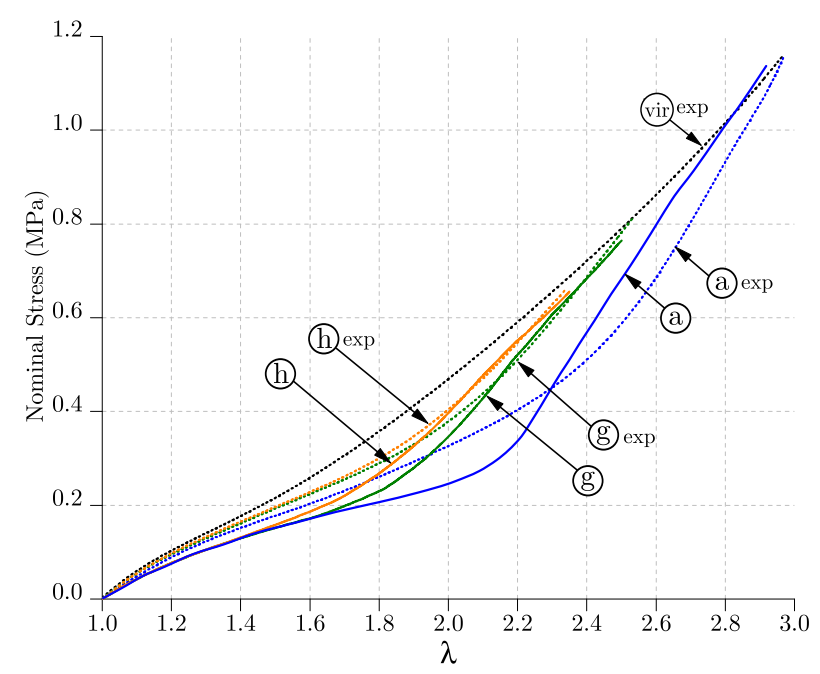

Figure 3: Comparison of the model (solid lines) with biaxial pre-stretching experimental data (dotted lines). Curve $a$ presents simulation of the model for the second load after an equibiaxial test; curve $g$ presents simulation of the model for the second load after a biaxial test of biaxiality rate $\mu=0.7$; curve $h$ presents simulation of the model for the second load after a biaxial test of biaxiality rate $\mu=0.5$ 
mentation needs a quasi-incompressible formulation (Federico et al., 2008; Holzapfel et al., 2000; Holzapfel and Gasser, 2001; Ogden, 2003, 1997; Sun W, 2005), the equation must be rewritten in terms of incompressible invariants as follows:

$$
\mathcal{W}=\overline{\mathcal{W}}\left(\bar{I}_{1}, \bar{I}_{2}, \bar{I}_{4}{ }^{(i)}\right)+\mathcal{U}(J)
$$

with $J^{2}=I_{3}=\operatorname{det}(\mathbf{C})$ is the volume variation, and $\bar{I}_{1}=I_{3}^{-1 / 3} I_{1}, \bar{I}_{2}=I_{3}^{-2 / 3} I_{2}, \bar{I}_{4}{ }^{(i)}=I_{3}^{-1 / 3} I_{4}^{(i)}$ are the incompressible invariants. The problem of the implementation of anisotropic hyperelastic energy densities has ever been tackled in literature, see for example (Peña et al., 2009; Bose and Dorfmann, 2009; Kroon and Holzapfel, 2008). After the decomposition given in Eq. 4, the second Piola-Kirchhoff stress tensor $\mathbf{S}$ is obtained by the strain energy derivation, as

$$
\mathbf{S}=\overline{\mathbf{S}}+\mathbf{S}^{v o l}=2\left\{\frac{\partial \overline{\mathcal{W}}}{\partial \bar{I}_{1}} \frac{\partial \bar{I}_{1}}{\partial \mathbf{C}}+\frac{\partial \overline{\mathcal{W}}}{\partial \bar{I}_{2}} \frac{\partial \bar{I}_{2}}{\partial \mathbf{C}}+\sum_{i=1}^{n} \frac{\partial \overline{\mathcal{W}}}{\partial \bar{I}_{4}{ }^{(i)}} \frac{\partial \bar{I}_{4}^{(i)}}{\partial \mathbf{C}}\right\}+J \frac{\partial \mathcal{U}}{\partial J} \mathbf{C}^{-1}
$$

Where $\overline{\mathbf{S}}$ and $\mathbf{S}^{\text {vol }}$ are respectively the isochoric and volumetric parts of the second Piola-Kirchhoff stress tensor $\mathbf{S}$. The lagrangian deviatoric tangent modulus, is finally given by

$$
\overline{\mathbb{C}}^{L}=4\left\{\frac{\partial}{\partial \mathbf{C}}\left(\overline{\mathcal{W}}, 1 \frac{\partial \bar{I}_{1}}{\partial \mathbf{C}}\right)+\frac{\partial}{\partial \mathbf{C}}\left(\overline{\mathcal{W}}, 2 \frac{\partial \bar{I}_{2}}{\partial \mathbf{C}}\right)+\sum_{i=1}^{n} \frac{\partial}{\partial \mathbf{C}}\left(\overline{\mathcal{W}}_{4}{ }_{4}^{(i)} \frac{\partial \bar{I}_{4}{ }^{(i)}}{\partial \mathbf{C}}\right)\right\}
$$

Where $\overline{\mathcal{W}},_{k}$ notes $\frac{\partial \bar{W}}{\partial I_{k}}$. The eulerian deviatoric modulus is deduced by means of the push-forward operation $\Phi_{*}$ as $\overline{\mathbb{C}}^{e}=\frac{1}{J} \Phi_{*}\left(\overline{\mathbb{C}}^{L}\right)$. Finally the stress and the tangent modulus are given by the first and second derivates of the strain energy function with respect to the first, second and fourth invariants. The general form of $\overline{\mathbb{C}}^{L}$ and $\mathbb{C}_{\text {vol }}^{L}$ are presented in Kaliske (2000). For the present model, the particular forms of the derivatives of the energy are expressed as:

$$
\begin{aligned}
\overline{\mathcal{W}}_{4}^{(i)} & =2 k w^{(i)} \mathcal{F}^{(i)}\left(\bar{I}_{4}^{(i)}-1\right) \\
\overline{\mathcal{W}}_{44}^{(i)} & =2 k w^{(i)}\left\{\frac{\partial \mathcal{F}^{(i)}}{\partial \bar{I}_{4}^{(i)}}\left(\bar{I}_{4}^{(i)}-1\right)+\mathcal{F}^{(i)}\right\} \\
\overline{\mathcal{W}} & { }_{14}^{(i)}=2 k w^{(i)} \frac{\partial \mathcal{F}^{(i)}}{\partial \bar{I}_{1}}\left(\bar{I}_{4}^{(i)}-1\right)
\end{aligned}
$$

with the following conditions

$$
\begin{aligned}
& \text { if } \quad \bar{I}_{1 \max }=I_{1} \quad \frac{\partial \mathcal{F}^{(i)}}{\partial \bar{I}_{1}}=0 \\
& \text { elseif } \quad \frac{\partial \mathcal{F}^{(i)}}{\partial \bar{I}_{1}}=\eta \frac{1}{2}\left(\frac{\bar{I}_{1 \max }-\bar{I}_{1}}{\bar{I}_{1 \max }-3}\right)^{-\frac{1}{2}}\left(\frac{1}{\bar{I}_{1 \max }-3}\right)\left(\frac{\bar{I}_{4 \max }^{(i)}-\bar{I}_{4}^{(i)}}{\bar{I}_{4 \max }^{(i)}-1}\right)\left(\frac{\bar{I}_{4 \max }^{(i)}}{\bar{I}_{4 \max }}\right)^{4} \\
& \text { if } \quad \bar{I}_{4 \max }^{(i)}=\bar{I}_{4}^{(i)} \quad \frac{\partial \mathcal{F}^{(i)}}{\partial \bar{I}_{4}^{(i)}}=0 \\
& \text { elseif } \quad \frac{\partial \mathcal{F}^{(i)}}{\partial \bar{I}_{4}^{(i)}} \quad=\eta \sqrt{\frac{\bar{I}_{1 \max }-\bar{I}_{1}}{\bar{I}_{1 \max }-3}}\left(\frac{1}{\bar{I}_{4 \max }^{(i)}-1}\right)\left(\frac{\bar{I}_{4 \max }^{(i)}}{\bar{I}_{4 \max }}\right)^{4}
\end{aligned}
$$

Finally, the model is implemented via a UMAT in the Finite Element code Abaqus and simulations can be realized. 


\section{Validation of the model on a structure calculus}

\subsection{Presentation of an experimental holey plate tension test}

In order to test the ability of the model to simulate adequately a complex structure, an experimental test was designed. A RTV-3428 silicone rectangular plate sample with $2 \mathrm{~mm}$ in thickness was molded using the same protocol presented in Machado et al. (2010). After molding, five holes were generated. The final dimensions of the holey plate are described in Fig. 4(a). The plate was put into a tensile device which allows to record the force undergone by the plate. The force measure is synchronised with a Stereo Digital Image Correlation (SDIC) system. The SDIC technique allows the determination of three dimensional surface displacements and strain fields of the plate (Machado et al., 2012a). Thus, under the incompressibility assumption, in each point of the plate, strain tensor is evaluated.

The aim of this paper is to observe the Mullins effect, so a cyclic tensile test is designed. The strain field of the plate is studied for a macroscopic engineering strain of $50 \%$ after different maximal engineering strain: $50 \%, 100 \%$ and $150 \%$. It is chosen to study particularly three paths in the

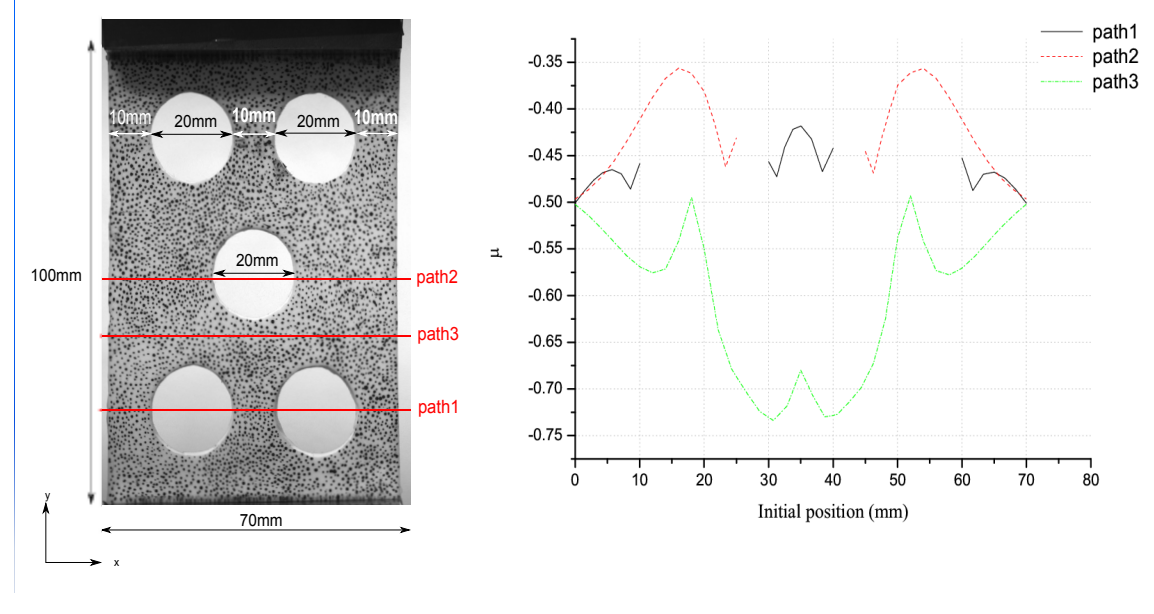

Figure 4: (a) Silicone plate sample containing five holes and paths of interest, (b) Biaxiality ratio along the three paths

holey plates, they are represented in Fig. 4(a). These paths were chosen in order to obtain very different deformation states, i.e., different values of biaxiality ratio $\mu$ : that allows to highlight the reliability of the proposed model. Fig. 4(b) presents the evolution of the biaxiality ratio at the same level of macroscopic deformation i.e. $50 \%$ for the three paths.

\subsection{Comparaison with model predictions}

Finite element simulations of the experiments were performed with the model in plane stress hypothesis with linear elements. The objective of this section is to compare the experimental and numerical results for an engineering strain of $50 \%$. The following notations are used in this part, $1 \mathcal{C}_{50}^{E F}$ and $1 \mathcal{C}_{50}^{\text {exp }}$ represent the first load for the numerical model and experimental results, respectively. In the same way, $2 \mathcal{C}_{100}^{E F}, 2 \mathcal{C}_{100}^{\exp }$, and $2 \mathcal{C}_{150}^{E F}, 2 \mathcal{C}_{150}^{\exp }$ represent the deformation at $50 \%$ after a first load of $100 \%$ and $150 \%$, respectively. 


\subsubsection{Global behavior of the plate}

The global displacement and the force undergone by the plate for the experimental and numerical tests are compared in Fig 5. It appears that the first and second loadings are very close. A small difference appears for the first loading but, it should be due to the underestimation of biaxial loading by the model. This small difference is only due to the hyperelastic constitutive equation and not to the stress softening function.

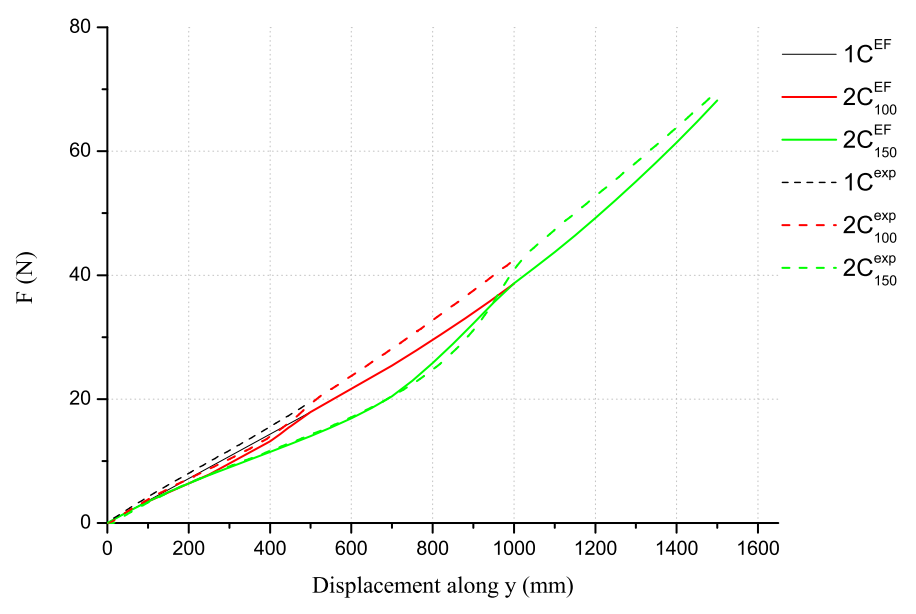

Figure 5: Comparison of global force response between the proposed model and the experimental holey plate.

\subsubsection{Contours of the plate and strain fields measurements}

Fig 6 presents the maximal principal logarithmic strain field obtained for the plate by finite element simulation and experimental measurement for $2 \mathcal{C}_{100}$ configurations. Considering the strain field distribution along the plate surface, it appears that the FE simulation represents quite well the experimental data. This is also valid for $1 \mathcal{C}$ and $2 \mathcal{C}_{150}$ configurations but these results are not presented in the paper. The contours of the plate are compared for $1 \mathcal{C}_{50}, 2 \mathcal{C}_{100}$ and $2 \mathcal{C}_{150}$ configuration and the results are presented in Fig 7 for a deformation of 50\%. The experimental tests are the gray picture and the FE simulations the contour lines in red. It is possible to observe that experimental results and numerical simulations are almost superposed.

\subsubsection{Observation of specific paths}

To focus on the local behavior of the plate, the maximal principal logarithmic strain along the different paths defined in Fig. 4 is presented in Fig. 8. A comparaison between the experimental and numerical tests is presented. According to the Fig. 8(a) the maximal principal logarithmic strain is greater for the second loadings than for the first loadings, but Fig. 8(b) and (c) show that the maximal principal logarithmic strain is more important for the first loadings that for the second loadings. This difference is explained by the local state of deformation reached along each path. The maximal deformation is reached along the path 1 so the stress-softening too. Since the stress-softening is maximal along the path1 it generates more important deformation for the along this same path seconds loadings. Along the paths 2 and 3 the stress-softening is smaller that along 


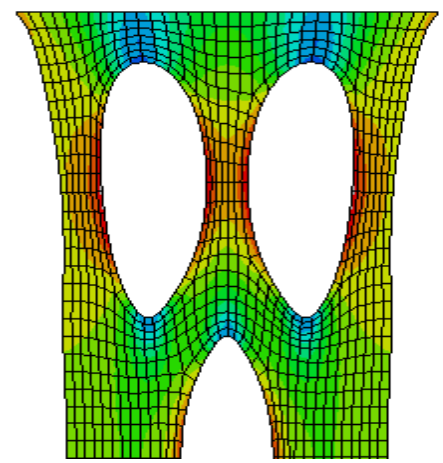

(a)

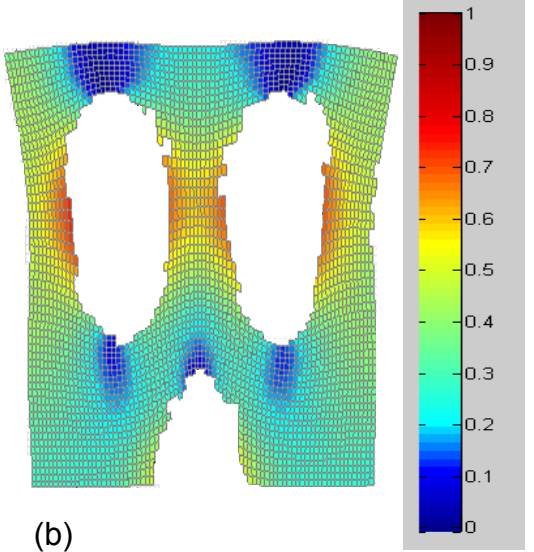

(b)

Figure 6: Maximal strain logaritmic fields of the silicone plate for the first loading : (a) numerical results and (b) experimental results.

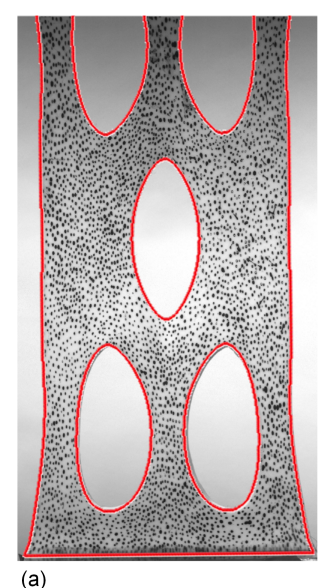

(a)

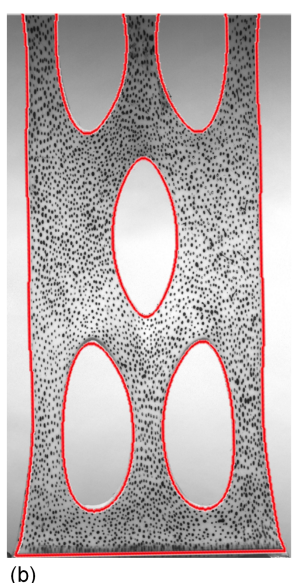

(b)

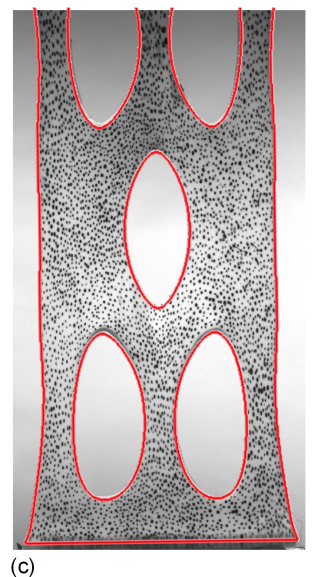

(c)

Figure 7: Comparison of the contours of the plate for $1 \mathcal{C}_{50}$ (a), $2 \mathcal{C}_{100}$ (b) and $2 \mathcal{C}_{150}$ (c) at $50 \%$ of deformation. Gray pictures represent exprimental data and red lines numerical data. 
the path1 so the deformations generated along these paths decrease for the second loadings since the global deformation of the holey is still the same as the first loading.

Fig. 8 also shows that the results are quite similar even if there is not a perfect spatial superposition between experimental and FE simulations. This difference can be also explained by the out-ofplane deformation, induced by the geometry of the plate, during the experimental test. Indeed for the experimental tests an out-of-plane displacement, close to the holes of the silicone plate, was observed. This out-of-plane displacement does not occur in numerical simulation since it is defined in plane stress. Thus, the superposition of the results is better along the path 2 (Fig. 8(b)) than along the paths 1 and 3 (cf. Fig. 8(a) and (c)) since the out-of-plane displacement observed is minimal near the hole of the path2 and maximal near the holes of paths 1 and 3 .
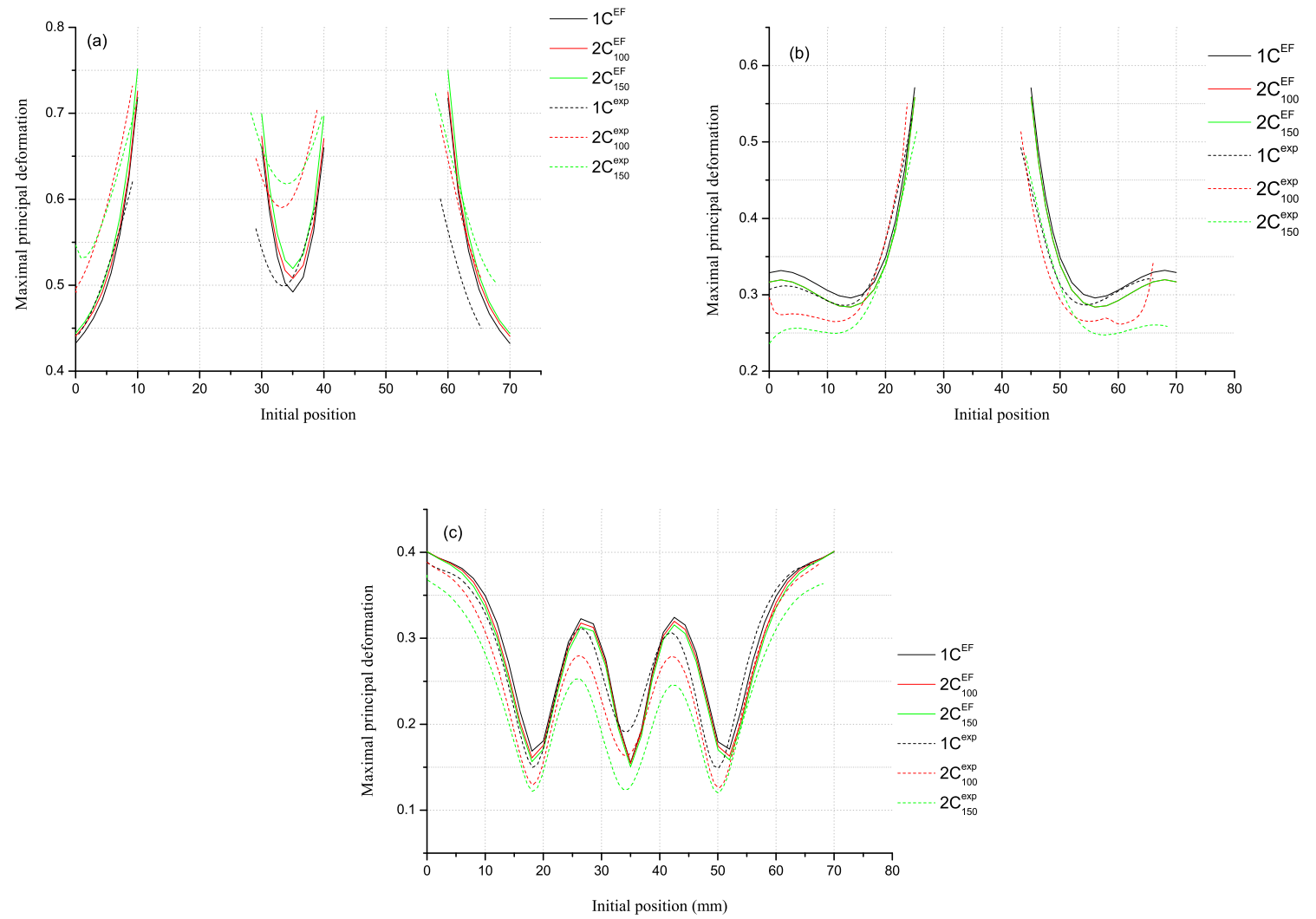

Figure 8: Maximal principal deformation for a global strain of 50\% along the path1 (a), the path2(b) and the path3 (c).

\section{Conclusion}

A new model was presented to take into account the anisotropy induced by stress softening during deformation of a rubber like material. The proposed form depends both of global maximal equivalent strain deformation and directinal maximal strains. The model was written using only 
strain invariants which permits to have a formulation well adapted to finite element implementation. Details about tangent modulus calculation were given in the paper.

The ability of the proposed model to simulate experimental data was tested first on classical tests (uniaxial, planar and equibiaxial tension) and second on tests where the loading directions were changing between first and second loadings. The analysis of a holey plate was realized by means of digital image correlation which allows to validate the global force-displacement response and local deformation of the plate following three different paths (with diffrent type of local strain). Finally, all the results highligt the good efficiency of the anisotropic stress-softening function which depends on only one parameter. This model can be used in finite element softwares. It allows to take account very particular loading paths.

\section{References}

Bazant, Z. P. and Oh, B. H. (1986). Efficient numerical integration on the surface of a sphere. Z. Angew. Math. Mech., 66, 37-49.

Besdo, D. and Ihlemann, J. (2003). Properties of rubber like materials under large deformations explained by self-organizing linkage patterns. Int. J. Plast., 19, 1001-1018.

Bose, K. and Dorfmann, A. (2009). Computational aspects of a pseudo-elastic constitutive model for muscle properties in a soft-bodied arthropod. Int. J. Non-Linear Mech., 44, 42-50.

Cantournet, S., Desmorat, R., and Besson, J. (2009). Mullins effect and cyclic stress softening of filled elastomers by internal sliding and friction thermodynamics model. Int. J. Solids Struct., 46, $2255-2264$.

Chagnon, G., Verron, E., Gornet, L., Marckmann, G., and Charrier, P. (2004). On the relevance of continuum damage mechanics as applied to the Mullins effect: theory, experiments and numerical implementation. J. Mech. Phys. Solids, 52, 1627-1650.

Chagnon, G., Verron, E., Marckmann, G., and Gornet, L., (2006). Development of new constitutive equations for mullins effect in rubber using the network alteration theory. Int. J. Solids Struct., 43, 6817-6831.

Dargazany, R. and Itskov, M. (2009). A network evolution model for the anisotropic Mullins effect in carbon black filled rubbers. Int. J. Solids Struct., 46, 2967-2977.

Diani, J., Brieu, M., and Vacherand, J. M. (2006a). A damage directional constitutive model for the Mullins effect with permanent set and induced anisotropy. Eur. J. Mech. A/Solids, 25, 483-496.

Diani, J., Brieu, M., and Gilormini, P. (2006b). Observation and modeling of the anisotropic visco-hyperelastic behavior of a rubberlike material. Int. J. Solids Struct., 43, 3044-3056.

Dorfmann, A., and Pancheri, F. Q. (2012). A constitutive model for the Mullins effect with changes in material symmetry. Int. J. Nonlinear Mech., 47, 874-887.

Federico, S., Grillo, A., Imatani, S., Giaquinta, G., and Herzog, W. (2008). An energetic approach to the analysis of anisotropic hyperelastic materials. Int. J. Eng. Sci., 46, 164-181.

Göktepe, S. and Miehe, C. (2005). A micro-macro approach to rubber-like materials. Part III: The micro-sphere model of anisotropic Mullins-type damage. J. Mech. Phys. Solids, 53, 2259-2283.

Govindjee, S. and Simo, J. C. (1992). Mullins' effect and the strain amplitude dependence of the storage modulus. Int. J. Solids. Structures, 29(14-15), 1737-1751.

Gracia, L. A., Peña, E., Royo, J. M., Pelegay, J. L., and Calvo, B. (2009). A comparison between pseudo-elastic and damage models for modelling the Mullins effect in industrial rubber components. Mech. Res. Comm., 36, 769-776.

Guo, Z. Y., Peng, X. Q., and Moran, B. (2006). A composites-based hyperelastic constitutive model for soft tissue with application to the human annulus fibrosus. J. Mech. Phys. Solids, 54, 1952-1971.

Hanson, D. E., Hawley, M., Houlton, R., Chitanvis, K., Rae, P., Orler, E. B., and Wrobleski, D. A. (2005). Stress softening experiments in silica-filled polydimethylsiloxane provide insight into a mechanism for the Mullins effect. Polymer, 46, 10989-10995.

Harwood, J. A. C., Mullins, L., and Payne, A. R. (1965). Stress softening in natural rubber vulcanizates. Part 2. stress softeningeffects in pure gum and filler loaded rubbers. J. Appl. Polym. Sci., 9, 3011-3021.

Holzapfel, G. A. and Gasser, T. C. (2001). A viscoelastic model for fiber-reinforced composites at finite strains: continuum basis, computational aspects and applications. Comput. Methods Appl. Mech. Engrg, 190, 4379-4403.

Holzapfel, G. A., Gasser, T. C., and Ogden, R. W. (2000). A new constitutive framework for arterial wall mechanics and a comparative study of material models. J. Elast., 61, 1-48. 
Itskov, M., Haberstroh, E., Ehret, A. E., and Vohringer, M. C. (2006). Experimental observation of the deformation induced anisotropy of the Mullins effect in rubber. KGK-Kautschuk Gummi Kunststoffe, 59(3), 93-96.

Itskov, M., Ehret, A., Kazakeviciute-Makovska, R., and Weinhold, G. (2010). A thermodynamically consistent phenomenological model of the anisotropic Mullins effect. ZAMM - J. Appl. Math. Mech., 90, 370-386.

Kaliske, M. (2000). A formulation of elasticity and viscoelasticity for fibre reinforced material at small and finite strains. Comput. Methods Appl. Mech. Engrg, 185, 225-243.

Kroon, M. and Holzapfel, G. A. (2008). A new constitutive model for multilayered collagenous tissues. J. Biomech., 41, 2766-2771.

Laraba-Abbes, F., Ienny, P., and Piques, R. (2003). A new Taylor-made methodology for the mechanical behavior analysisof rubber like materials: II. Application of the hyperelastic behavior characterization of a carbon-black filled natural rubber vulcanizate. Polymer, 44, 821-840.

Machado, G., Chagnon, G., and Favier, D. (2010). Analysis of the isotropic models of the Mullins effect based on filled silicone rubber experimental results. Mech. Mater., 42, 841-851.

Machado, G., Favier, D., and Chagnon, G. (2012a). Determination of membrane stress-strain full fields of bulge tests from SDIC measurements. Theory, validation and experimental results on a silicone elastomer. Exp. Mech., 52 865-880.

Machado, G., Chagnon, G., and Favier, D. (2012b). Induced anisotropy by the mullins effect in filled silicone rubber. Mech. Mater., 50, $70-80$.

Marckmann, G. and Verron, E. (2006). Comparison of hyperelastic models for rubber-like materials. Rubber Chem. Technol., 79, 835-858.

Marckmann, G., Verron, E., Gornet, L., Chagnon, G., Charrier, P., and Fort, P. (2002). A theory of network alteration for the Mullins effect. J. Mech. Phys. Solids., 50, 2011-2028.

Merckel, Y.,Diani, J., Roux, S., and Brieu, M. (2011). A simple framework for full-network hyperelasticity and anisotropic damage. J. Mech. Phys. Solids., 59, $75-88$.

Merckel, Y., Brieu, M., Diani, J., and Caillard, J. (2012). A Mullins softening criterion for general loading conditions. J. Mech. Phys. Solids., 60, $1257-1264$.

Meunier, L., Chagnon, G., Favier, D., Orgéas, L., and Vacher, P. (2008). Mechanical experimental characterisation and numerical modelling of an unfilled silicone rubber. Polym. Test., 27, 765-777.

Miehe, C. and Keck, J. (2000). Superimposed finite elastic-viscoelastic-plastoelastic stress response with damage in filled rubbery polymers. experiments, modelling and algorithmicimplementation. J. Mech. Phys. Solids, 48, 323-365.

Miehe, C., Göktepe, S., and Lulei, F. (2004). A micro-macro approach to rubber-like materials - part i: the nonaffine-micro-sphere model of rubber elasticity. J. Mech. Phys. Solids, 52, 2617-2660.

Mooney, M. (1940). A theory of large elastic deformation. J. Appl. Phys., 11, 582-592.

Muhr, A. H., Gough, J., and Gregory, I. H. (1999). Experimental determination of model for liquid silicone rubber: Hyperelasticity and Mullins effect. In Proceedings of the First European Conference on Constitutive Models for Rubber, pages 181-187.

Ogden, R. W. (1997). Non-linear Elastic Deformations. Dover Publications, New York.

Ogden, R. W. (2003). Nonlinear elasticity, anisotropy, material stability and residual stresses in soft tissue., chapter Holzapfel GA, Ogden RW (eds) Biomechanics of soft tissue in cardiovascular system. CISM Courses and lecture Series, vol 441. Springer, Wien New York.

Park, B. H. and Hamed, G. R. (2000). Anisotropy in gum and black filled SBR and NR vulcanizates due to large deformation. Korea Polym. J., 8, 268-275.

Pawelski, H. (2001). Softening behavior of elastomeric media after loading in changing directions. Constitutive models for rubber, Besdo, Schuster \& Ihleman (eds), pages 27-34.

Peña, E., Peña, J. A., and Doblar, M. (2009). On the Mullins effect and hysteresis of fibered biological materials: A comparison between continuous and discontinuous damage models. Int. J. Solids Struct., 46, 17271735.

Shariff, M. H. B. M. (2006). An anisotropic model of the Mullins effect. J. Eng. Math., 56, 415-435.

Sun W, S. M. (2005). Finite element implementation of a generalized Fung-elastic constitutive model for planar soft tissues. Biomech Model Mechanobiol.

Wu, P. D. and Van der Giessen, E. (1993). On improved network models for rubber elasticity and their applications to orientation hardening in glassy polymers. J. Mech. Phys. Solids., 41, 427-456.

Zuñiga, A. E. and Beatty, M. F. (2002). A new phenomenological model for stress-softening in elastomers. Z. Angew. Math. Phys., 53, 794-814. 